\title{
Increasing Output Power of Electromagnetic Vibration Energy Harvesters using Improved Halbach Arrays
}

\author{
Dibin Zhu ${ }^{1}$, Steve Beeby, John Tudor and Nick Harris \\ School of Electronics and Computer Science, University of Southampton, Southampton, \\ SO17 1BJ, UK
}

\begin{abstract}
This paper extends previously published studies into the performance of Halbach arrays for electromagnetic vibration energy harvesting. A Halbach array is a specific arrangement of permanent magnets that concentrates the magnetic field on one side of the array while cancelling the field to almost zero on the other side. This arrangement can improve electromagnetic coupling in a limited space. Previous research showed that although the Halbach array has higher magnetic field density compared to normal magnet layouts, its magnetic flux change rate is not necessarily high. Thus, output powers of energy harvesters with standard Halbach arrays are not always greater than those with normal magnet layouts. Two improvements to the Halbach arrays that lead to increased output power of electromagnetic vibration energy harvesters are presented in this paper. Test results showed that the proposed improved Halbach arrays can increase the output power of energy harvesters by a factor of seven compared to the standard Halbach array.
\end{abstract}

Keywords: Electromagnetic energy harvester, Halbach array

\section{Introduction}

Vibration energy harvesting offers promise as a solution for powering wireless sensor nodes, and has been studied comprehensively in recent years. Piezoelectric, electromagnetic, electrostatic and magnetostrictive transduction mechanisms are commonly used to convert mechanical energy into electrical energy [1]. Among these transducers, the electromagnetic transducer has received special attention due to its high power density, especially at the macro-scale. Methods have been developed to increase the output power of electromagnetic energy harvesters, such as optimising the power conditioning circuitry [2], using adaptive energy harvesters [3] and using different magnet layouts, such as Halbach arrays [4].

A Halbach array (HA) is a specific arrangement of permanent magnets that concentrates the magnetic field on one side of the array while cancelling the field to almost zero on the other side. It consists of two sets of magnets, i.e. main magnets and transit magnets as shown in Figure 1. The superposition of the magnetic flux caused by the main magnets and the transit magnets concentrates the magnetic field to one side of the Halbach array (the active side). Assuming the magnetic field strength generated by the main magnets is identical to that generated by the transit magnets, the active-side of the Halbach array will have double the magnetic field strength compared with the normal configuration. The other side of the Halbach array (the quiet-side) will have a negligible magnetic field.

\footnotetext{
${ }^{1}$ Corresponding author, Email: dz@ecs.soton.ac.uk
} 

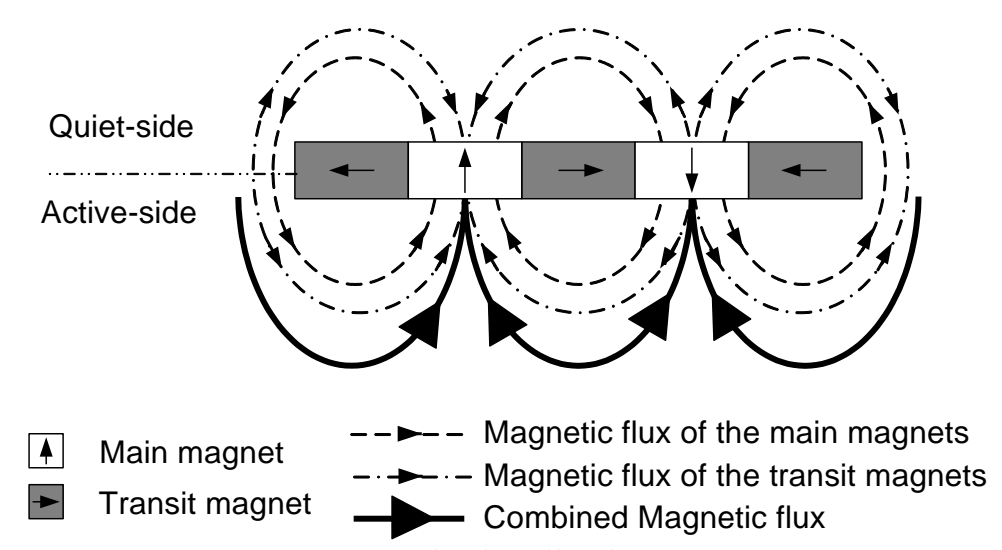

(a) Standard Halbach array

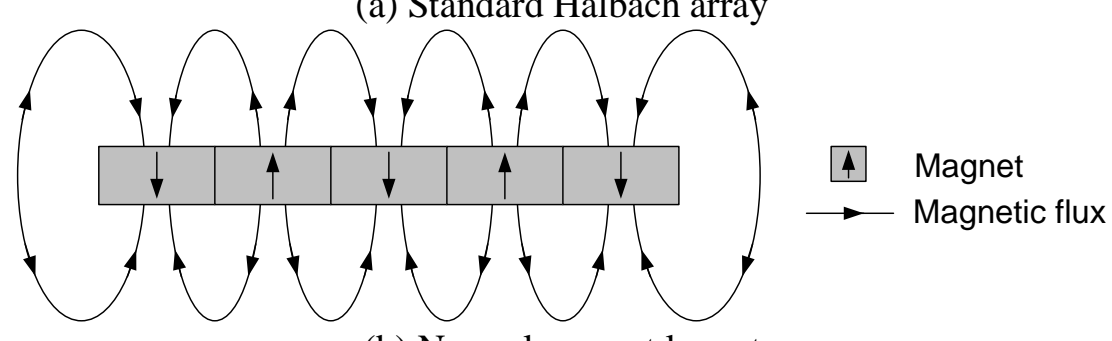

(b) Normal magnet layout

Figure 1. Halbach arrays and normal magnet layout.

There are two types of Halbach arrays, i.e. flat Halbach arrays and Halbach cylinders as shown in Figure 2. Flat Halbach arrays have applications such as wiggler magnets used in particle accelerators and free electron lasers [5] and linear motors [6]. Applications of Halbach cylinders include brushless AC motors [7], magnetic coupling and high field cylinders [8] and linear alternators [9].

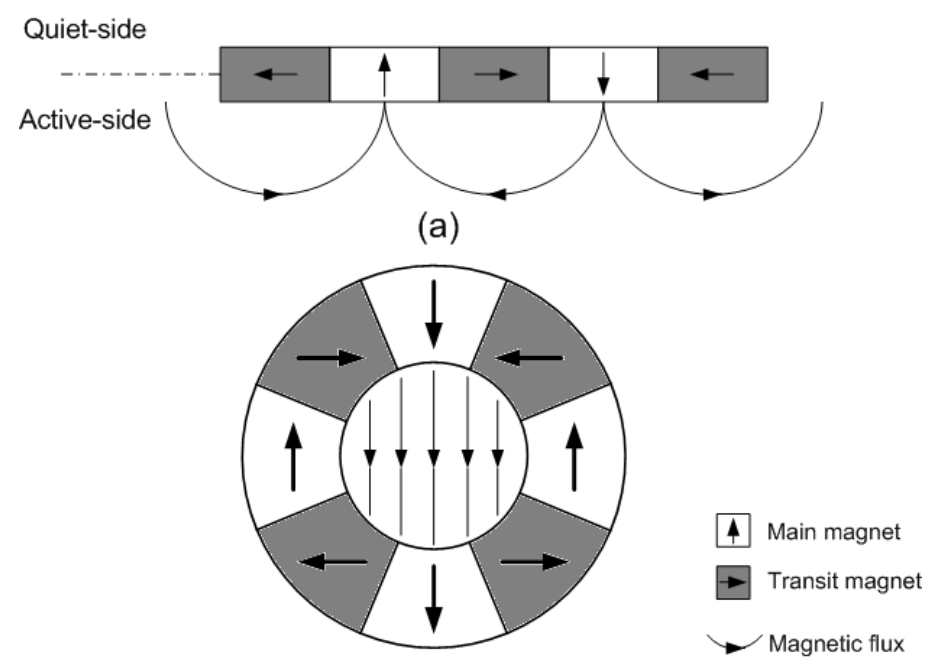

(b)

Figure 2. (a) Flat Halbach array and (b) Halbach cylinders.

The application of Halbach arrays in vibration energy harvesting has recently been reported. Wang et al [10] reported an energy harvester consisting of a circular Halbach array and the authors of this paper [4] presented the use of a flat Halbach array in energy harvesting. Experimental results in [4] showed that although the Halbach array has higher magnetic field density compared to normal magnet layouts, its magnetic flux change rate is not as high. Thus, output powers of energy harvesters with standard Halbach arrays are not always greater than those with normal magnet layouts. The reason for this is that the existence of transit magnets means that the magnet set has to move a long distance before the magnetic flux though the coil changes significantly, which makes it hard to achieve a high 
magnetic flux change rate. As a result, the output power of the energy harvester with a Halbach array is lower than generators with some normal magnet layouts due to their greater magnetic flux change rate, despite the smaller magnetic field strength.

This paper presents improvements of Halbach arrays in vibration energy harvesting. The main target is to minimise the effect of the transit magnets and achieve high magnetic flux change rate, thus increasing output power without significantly increasing the number of magnets in the magnetic arrangement compared to a standard Halbach array presented in [4]. Two designs of electromagnetic energy harvesters with improved Halbach arrays and their resulting output powers are discussed and compared to those of the previous design.

\section{Overview}

The principle of operation of an electromagnetic vibration energy harvester with a Halbach array is illustrated in Figure 3. The Halbach array is suspended by a spring structure. The coil is placed on the active side of the Halbach array and fixed to the housing of the energy harvester. Relative motion between the coil and the Halbach array generates electrical current. Unlike most existing vibration energy harvesters, this energy harvester has an in-plane displacement, which allows more space for the resonator to move within a low-profile structure. Figure 4(a) shows the assembled planar energy harvester. The overall dimensions are $55 \mathrm{~mm} \times 55 \mathrm{~mm} \times 4 \mathrm{~mm}$. Coils were placed underneath the Halbach array with a $0.5 \mathrm{~mm}$ gap in-between. Dimensions of specific coils used in tests will be presented later in Section 3 and 4. Figure 4(b) shows the setup of the energy harvester on a shaker. All tests described in this paper were done on this platform.

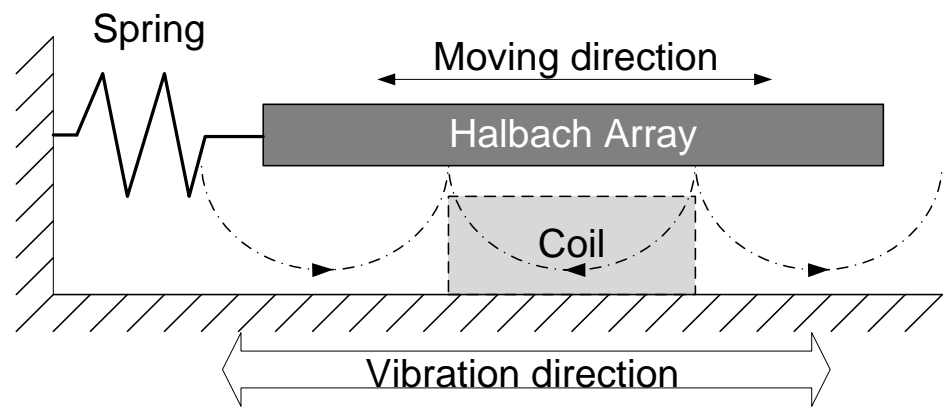

Figure 3. Structure of the energy harvester with a Halbach array.

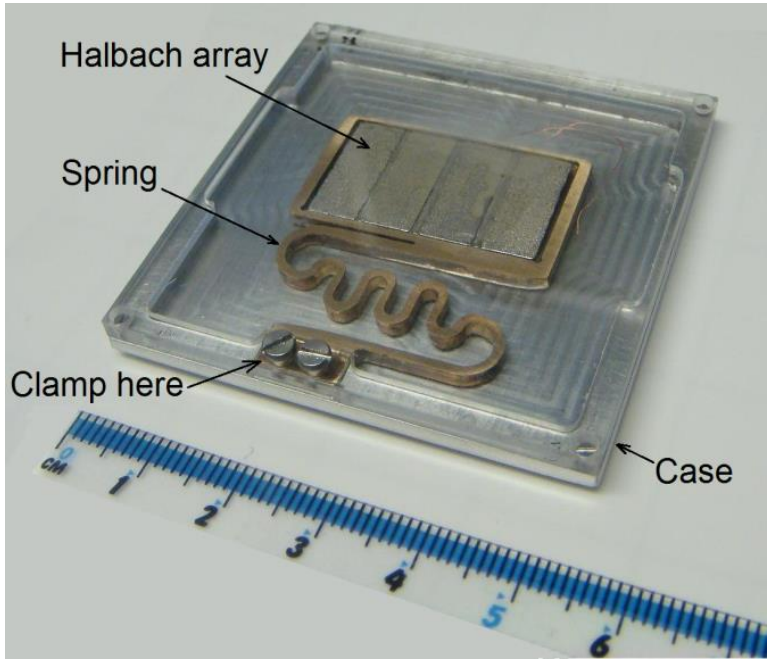

(a) Assembled energy harvester

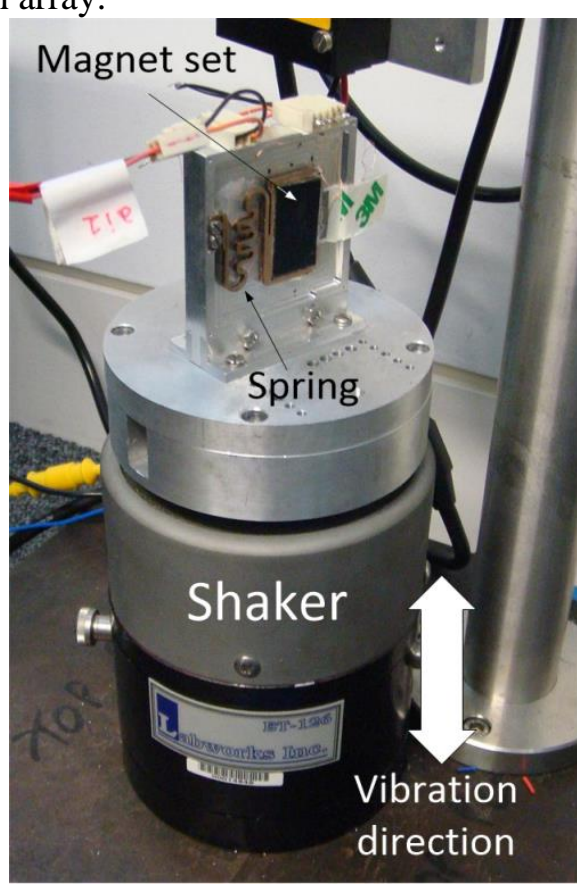

(b) Test setup

Figure 4. Assembled energy harvester and test setup (Coils are located under the Halbach Array). 


\section{Improvement 1: Halbach array consisting of magnets with triangular cross sections}

\subsection{Structure}

In order to minimise the effect of the transit magnets in a standard Halbach array and increase the magnetic flux change rate, magnets with triangular cross sections were investigated. The overall structure is shown in Figure 5(a). Its dimensions and magnetisation are shown in Figure 5(b). Each magnet is $16 \mathrm{~mm}$ long and $7 \mathrm{~mm}$ wide.

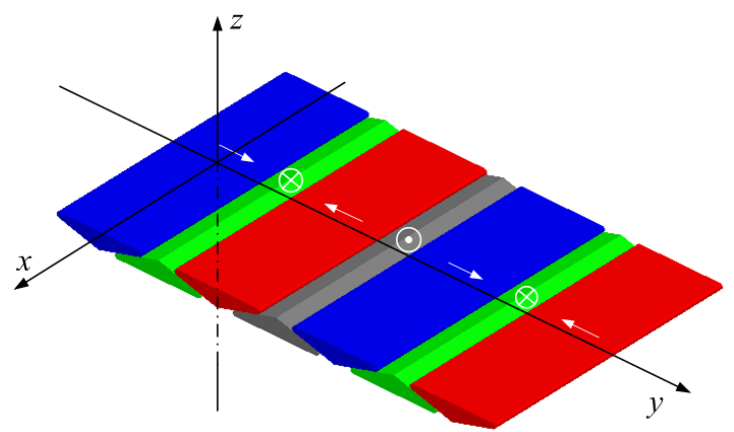

(a)

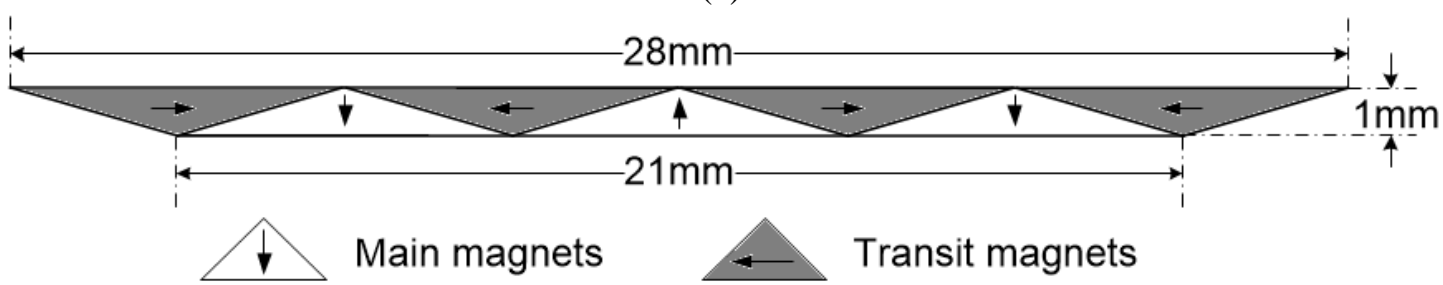

(b)

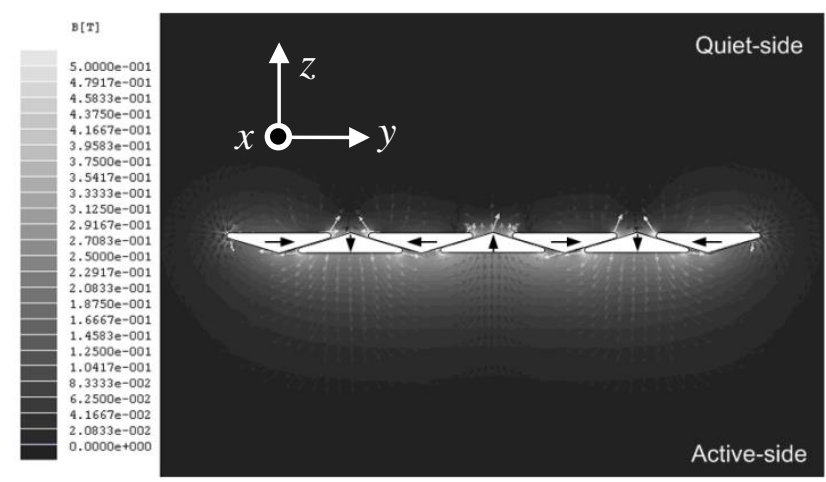

(c)

Figure 5. Halbach array consisting of magnets with triangular cross sections (a) structure (b) cross section view from the $y z$ plane (c) simulation results of magnetic field.

Figure 5(c) shows the magnetic field simulation results for the Halbach array with triangular crosssectioned magnets. It was found that there is an improved suppression of the magnetic field on the quiet side compared with that in a standard Halbach array with the same number of magnets as shown in Figure 6(b), while the field on the active side is distributed more evenly. Furthermore, magnetic forces between adjacent magnets are attractive while those in a standard Halbach array are repelling. Therefore, from a practical viewpoint, it is much easier to assemble the Halbach array with triangular cross-sectioned magnets. 


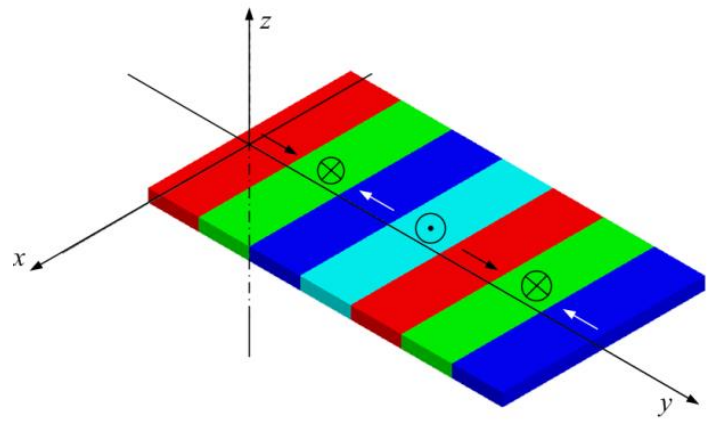

(a)

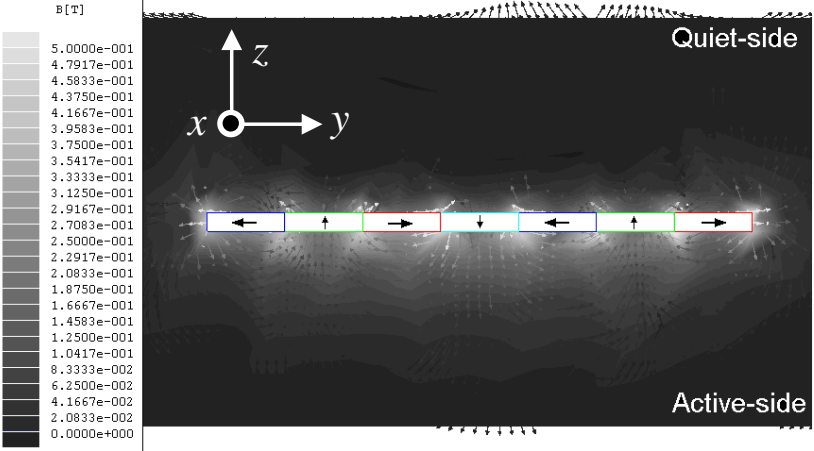

(b)

Figure 6. Standard Halbach array consisting of rectangular magnets (a) structure (b) simulation results of magnetic field.

To compare the performance of the Halbach array with triangular cross-sectioned magnets, a normal magnet layout with 7 magnets as shown in Figure 7(a) was used in the test as a comparison. This structure was found to have greater magnetic flux change rate and thus higher output power than a standard Halbach array as described in previous research [4]. To avoid confusion, it is worth mentioning that "normal layout" throughout this paper means magnet layout that only consists of magnets whose magnetization are along $z$ direction. The same spring frame and coil arrangement as used in [4] (Figure 8) was used in this test. The two $12 \mathrm{~mm}$ diameter, $1 \mathrm{~mm}$ thick coils were placed $0.5 \mathrm{~mm}$ beneath the magnet sets.

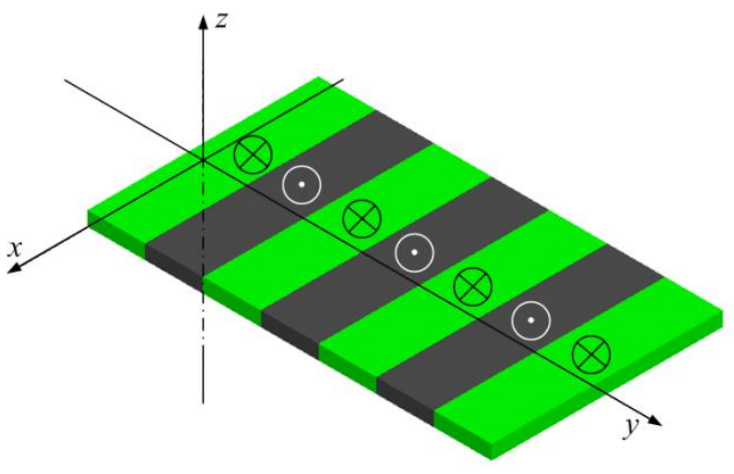

(a)

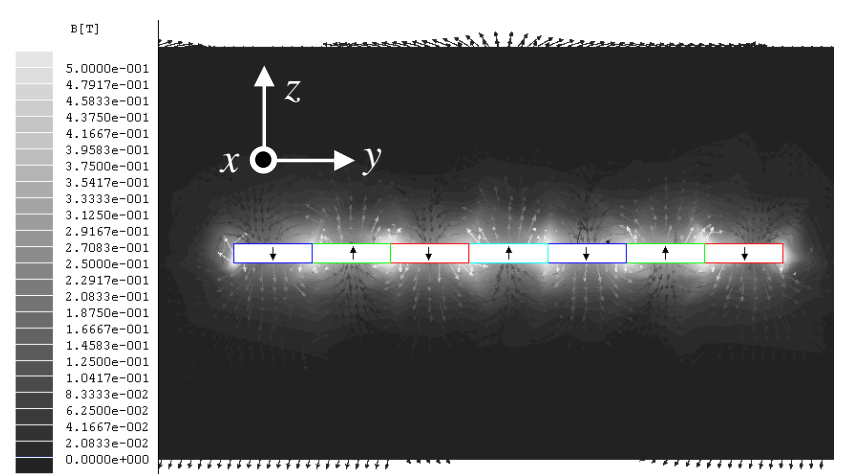

(b)

Figure 7. Normal magnet layout (7 magnets) (a) structure (b) simulation results of magnetic field.

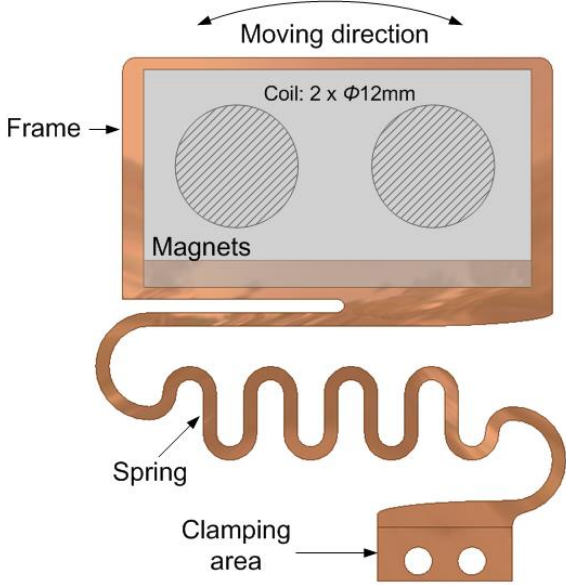

Figure 8. Energy harvester structure for the test of Halbach array with triangular cross-sectioned magnets. 


\subsection{Test results}

Table 1 compares the measured magnetic field density (B) in different locations in the three layouts. The magnetic field was measured by a gauss meter (GMET H001 from Magnet Sales and Service Limited). The probe has a Hall effect sensor with an active circular area of $2.5 \mathrm{~mm}$ in diameter. It was placed in planes parallel to the $x y$ plane and $\mathrm{Z} \mathrm{mm}$ beneath the bottom of the magnets. The maximum measured value in each case was recorded. It is found that at a distance of $1 \mathrm{~mm}$ below the magnets (the point at which the coils are located), the Halbach array with triangular cross-sectioned magnets has the highest magnetic field strength of the three test cases.

Table 1. Comparisons of measured magnetic field strength in different layouts of magnets (I).

\begin{tabular}{cccc}
\hline \multirow{2}{*}{ Layout of magnets } & \multicolumn{3}{c}{ Magnetic field strength (T) measured Z mm from the magnets } \\
\cline { 2 - 4 } & $\mathbf{Z = 0}$ & $\mathbf{Z = 0 . 5}$ & $\mathbf{Z = 1}$ \\
\hline Standard Halbach array & 0.26 & 0.21 & 0.14 \\
HA with triangular cross- & 0.225 & 0.17 & 0.145 \\
sectioned magnets & 0.21 & 0.158 & 0.136 \\
Normal layout (7 magnets) & &
\end{tabular}

The magnetic flux change rate in each case was then estimated in the plane $1 \mathrm{~mm}$ from the magnets using the following equation,

$$
\frac{d \varphi}{d t}=\frac{d\left(\int B \cdot d S\right)}{d t}=\frac{d\left(\int B \cdot w \cdot d D\right)}{d t}
$$

where $B$ is the magnetic field strength, $S$ is the area of the magnetic field, $w$ is the width of the entire magnet set $(16 \mathrm{~mm})$ and $D$ is the displacement. It was then normalised to the magnetic flux change rate of the standard Halbach array. The maximum tip displacement is $10 \mathrm{~mm}$ and the operational frequency is $49 \mathrm{~Hz}$.

Table 2 compares the magnetic flux change rate in different layouts of magnets. It was found that although the Halbach array with triangular cross-sectioned magnets almost doubles the magnetic flux change rate of the standard Halbach array, its magnetic flux change rate is still lower than that of the 7-magnet normal layout.

Table 2. Comparisons of magnetic flux change rate in different layouts of magnets (I).

\begin{tabular}{ccc}
\hline \multirow{2}{*}{ Layout of magnets } & \multicolumn{2}{c}{ Magnetic flux change rate } \\
\cline { 2 - 3 } & $\begin{array}{c}\text { Magnetic flux change rate } \\
(\mathbf{W b} / \mathbf{s})\end{array}$ & $\begin{array}{c}\text { Normalised magnetic flux change } \\
\text { rate }\end{array}$ \\
\hline Standard Halbach array & 0.001098 & 1 \\
HA with triangular cross- & 0.002063 & 1.88 \\
sectioned magnets & 0.002976 & 2.71 \\
Normal layout (7 magnets) &
\end{tabular}

All energy harvesters were excited with a sinusoidal vibration of $49 \mathrm{~Hz}$, subjected to accelerations between 0.1 and $0.5 \mathrm{G}_{\mathrm{rms}}$ whilst connected to their respective optimum resistive loads. Figure 9 shows the experimental comparisons of open circuit voltage and average output power of the energy harvesters at resonance with the different magnet layouts and coils. It was found that the output powers of energy harvesters with HA with triangular cross-sectioned magnets and 7-magnet normal layout are three and seven times greater than that of the standard Halbach array respectively. As the ratio of the flux change rate of the three arrangements is $1: 1.88: 2.71$, the theoretical ratio of power is $1^{2}: 1.88^{2}: 2.71^{2}=1: 3.52: 7.34$ and this shows good agreement with the ratio found experimentally as shown in Figure 9(b). 


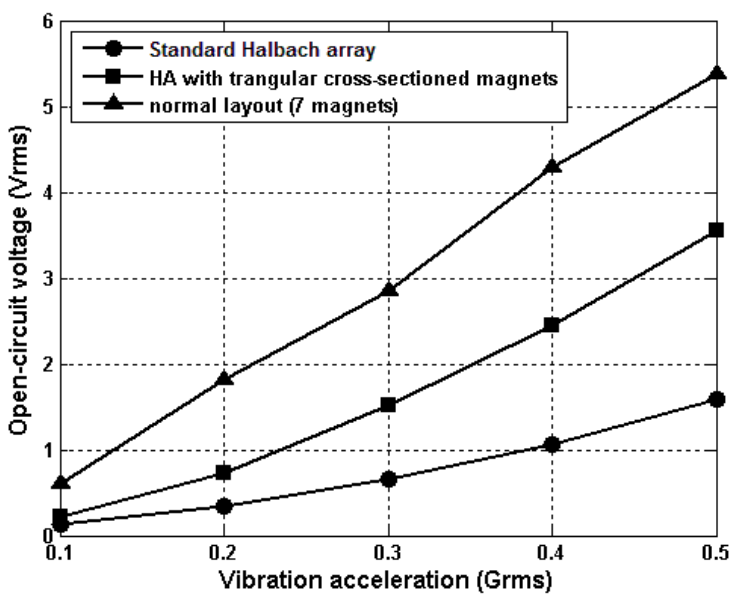

(a)

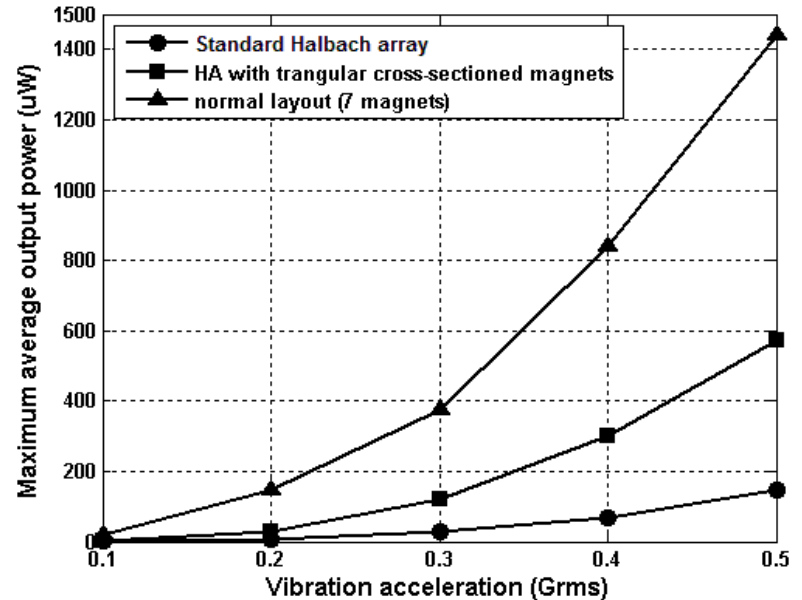

(b)

Figure 9. Comparisons of test results of standard Halbach array, Halbach array with triangular crosssectioned magnets and normal layouts (7 magnets) (a) open circuit voltage (b) maximum average output power.

\section{Improvement 2: double Halbach array}

\subsection{Structure}

A double Halbach array shown in Figure 10(a) was also investigated. It consists of two identical Halbach arrays that are placed side by side with the polarity of the magnets flipped. Both arrays have their active sides in the negative $z$ direction.

The magnetic field simulation results are shown in Figures 10(b) and 10(c). For each Halbach array, the magnetic flux is concentrated on one side of the magnets (negative $z$ direction) which is the same as a standard Halbach array as shown in Figure 1(a). By observing the magnetic flux distribution along the $y$ axis, it was found that the magnetic flux direction reverses immediately across the transition line. If coils are placed beneath the transition line and the Halbach array moves in the $y$ direction, a higher magnetic flux change rate, and thus increased output power can be achieved. 


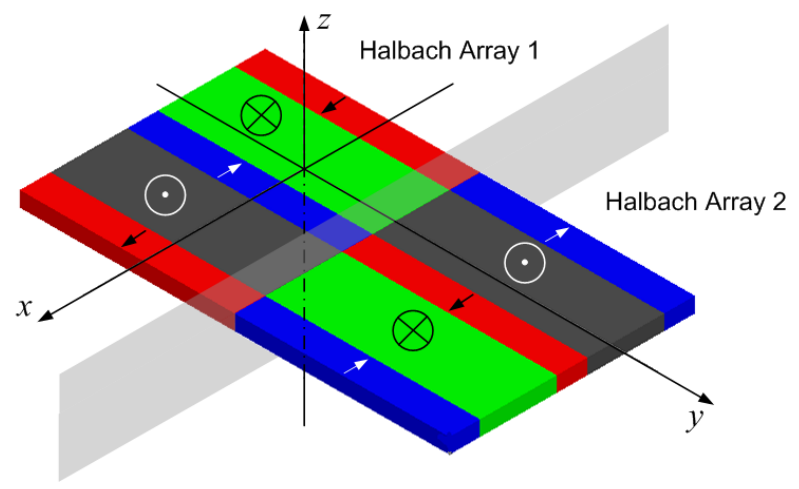

(a)

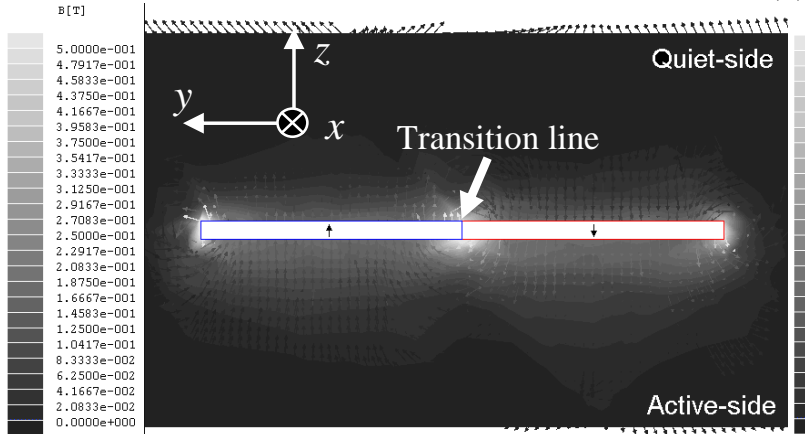

(b)

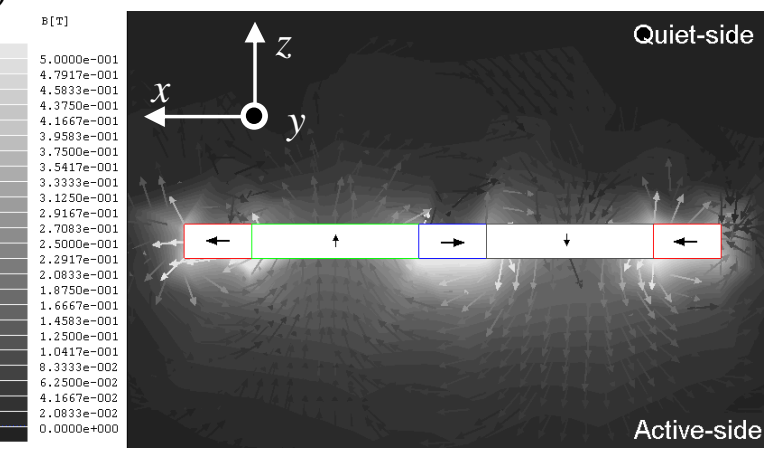

(c)

Figure 10. Double Halbach array (a) structure (b) simulation results of magnetic field in $y z$ plane (Double Halbach Array) (c) simulation results of magnetic field in $x z$ plane (Double Halbach Array).

The double Halbach array was compared with three other magnet layouts that occupy the same total area $\left(28 \times 16 \times 1 \mathrm{~mm}^{3}\right)$ : normal 4 magnets (Figure 11(a)), standard Halbach array (Figure 6(a)) and normal 7 magnets (Figure 7(a)). The normal 4 magnets layout was chosen because it has a similar magnetic flux distribution to that of the double Halbach array in the negative $z$ direction. The normal 7 magnets layout was chosen as it was found to have the maximum magnetic flux change rate in the previously reported test [4]. 


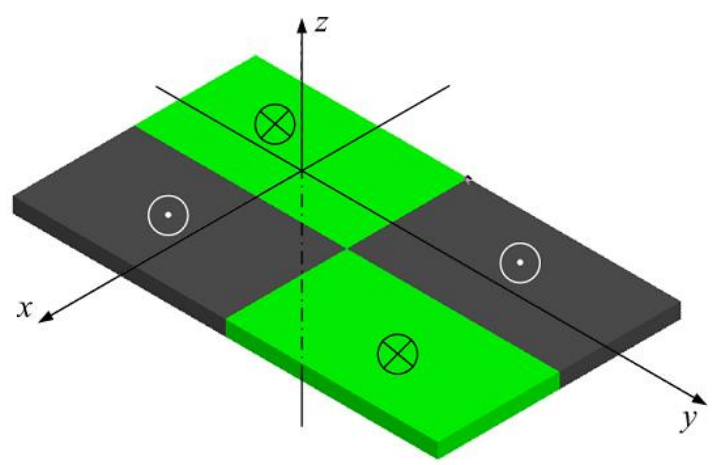

(a)

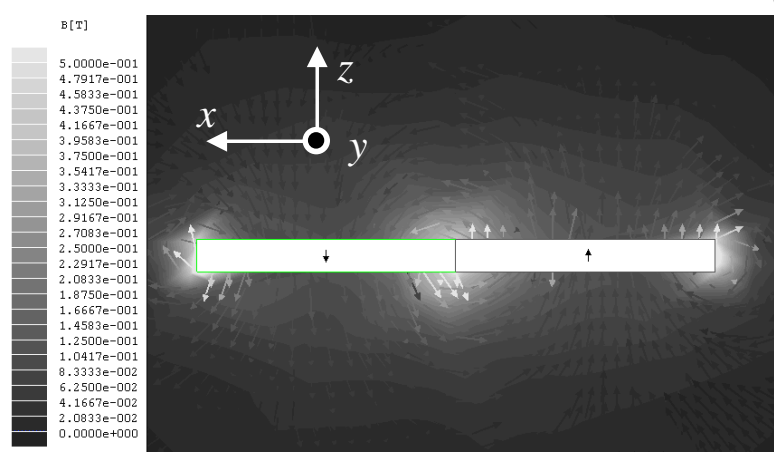

(b)

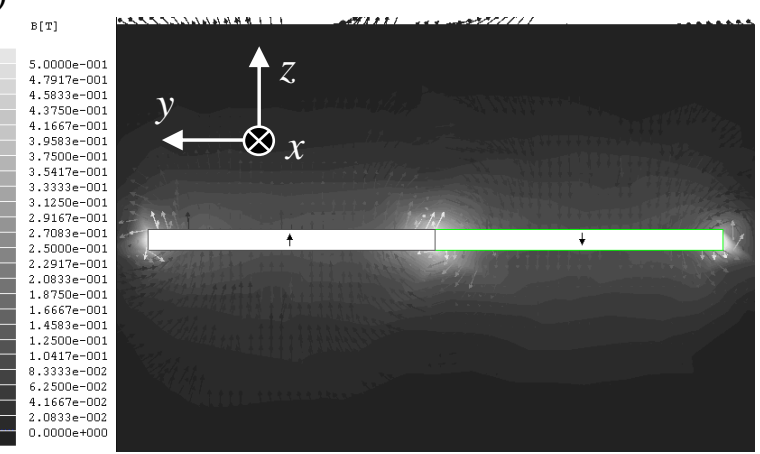

(c)

Figure 11. Normal four-magnet layout (a) structure (b) simulation results of magnetic field in $y z$ plane (Normal four-magnet layout) (c) simulation results of magnetic field in $x z$ plane (Normal fourmagnet layout).

Each of the magnet sets was attached to a meander spring made of $\mathrm{BeCu}$ as shown in Figure 12. The meander spring allows the resonator to move in-plane ( $y$ direction) with a low resonant frequency. The spring was designed to be stiffer in the $x$ and $z$ directions increasing the resonant frequencies of modes in these directions beyond the range of interest. The frame connected to the spring holds the Halbach array and additional mass. The resonant frequency of the resonator can be adjusted by varying the mass before installation.

Two coil arrangements were investigated for comparison. The first has one coil with a diameter of 11 $\mathrm{mm}$ and a thickness of $1 \mathrm{~mm}$. The coil resistance was $1620 \Omega$. The second arrangement has two small coils; each $6 \mathrm{~mm}$ in diameter and $1 \mathrm{~mm}$ thick. The two coils were connected in series and the total resistance was $1370 \Omega$. All coils were placed beneath the magnets with a $0.5 \mathrm{~mm}$ gap in-between. 


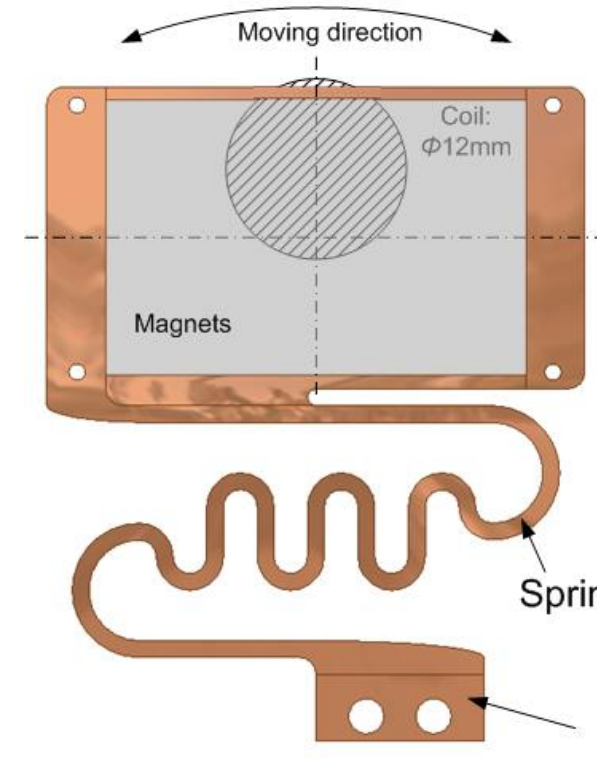

(a)
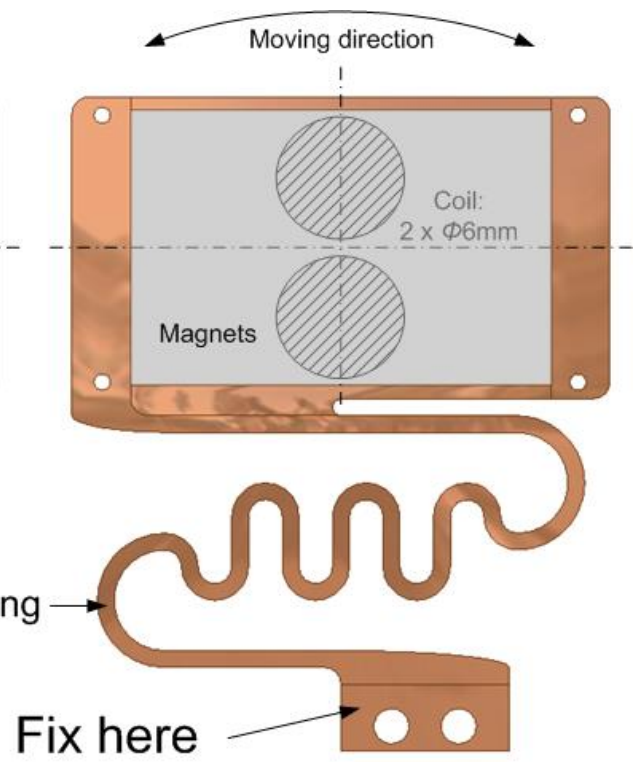

(b)

Figure 12. Energy harvester structure for the test of double Halbach array with (a) one big coil (b) two small coils.

\subsection{Test results}

Table 3 compares the measured magnetic field density (B) in different locations in the four layouts. The measurement was taken in planes parallel to the $x y$ plane and $\mathrm{Z} \mathrm{mm}$ beneath the bottom of the magnets. The maximum measured value in each case was recorded. It is found that, in the centre plane of the coils ( $1 \mathrm{~mm}$ from the magnet surface), the double Halbach array has the highest magnetic field strength.

Table 3. Comparisons of measured magnetic field strength in different layouts of magnets (II).

\begin{tabular}{cccc}
\hline \multirow{2}{*}{ Layout of magnets } & \multicolumn{3}{c}{ Magnetic field strength (T) measured $\mathbf{Z ~ m m}$ from the magnets } \\
\cline { 2 - 4 } & $\mathbf{Z = 0}$ & $\mathbf{Z = 0 . 5}$ & $\mathbf{Z = 1}$ \\
\hline Standard Halbach array & 0.26 & 0.21 & 0.14 \\
Double Halbach array & 0.185 & 0.17 & 0.153 \\
Normal layout (4 magnets) & 0.135 & 0.122 & 0.11 \\
Normal layout (7 magnets) & 0.21 & 0.158 & 0.136 \\
\hline
\end{tabular}

The magnetic flux change rate was then estimated in the plane $1 \mathrm{~mm}$ from the magnets using Eq. (1) and normalised to the magnetic flux change rate of the standard Halbach array. It was assumed that displacements $(10 \mathrm{~mm})$ and operational frequencies $(49 \mathrm{~Hz})$ are same in all cases.

Table 4. Comparisons of magnetic flux change rate in different layouts of magnets (one big coil).

\begin{tabular}{ccc}
\hline \multirow{2}{*}{ Layout of magnets } & \multicolumn{2}{c}{ Magnetic flux change rate } \\
\cline { 2 - 3 } & $\begin{array}{c}\text { Magnetic flux change rate } \\
(\mathbf{W b} / \mathbf{s})\end{array}$ & $\begin{array}{c}\text { Normalised magnetic flux change } \\
\text { rate }\end{array}$ \\
\hline Standard Halbach array & 0.001098 & 1 \\
Double Halbach array & 0.003009 & 2.74 \\
Normal layout (4 magnets) & 0.002339 & 2.13 \\
Normal layout (7 magnets) & 0.002416 & 2.2 \\
\hline
\end{tabular}


Table 5. Comparisons of magnetic flux change rate in different layouts of magnets (two small coils).

\begin{tabular}{ccc}
\hline \multirow{2}{*}{ Layout of magnets } & \multicolumn{2}{c}{ Magnetic flux change rate } \\
\cline { 2 - 3 } & $\begin{array}{c}\text { Magnetic flux change rate } \\
(\mathbf{W b} / \mathbf{s})\end{array}$ & $\begin{array}{c}\text { Normalised magnetic flux change } \\
\text { rate }\end{array}$ \\
\hline Standard Halbach array & 0.001098 & 1 \\
Double Halbach array & 0.003009 & 2.74 \\
Normal layout (4 magnets) & 0.002339 & 2.13 \\
Normal layout (7 magnets) & 0.002976 & 2.71 \\
\hline
\end{tabular}

Figure 4 and 5 compares magnetic flux change rate in different layouts of magnets in the case of one big coil and two small coils, respectively. The double Halbach array has the highest magnetic flux change rate in both cases and an increment by a factor of 2.74 was achieved compared to the standard Halbach array.

All energy harvesters were excited with a sinusoidal vibration of $49 \mathrm{~Hz}$ and subjected to accelerations between 0.1 and $0.6 \mathrm{G}_{\mathrm{rms}}$ and connected to their respective optimum resistive loads. Figure 13 and 14 show the experimental comparisons of output power of energy harvesters with different magnet layouts in one big coil and in two small coils, respectively. For the one-coil scenario, it was found that the output power of the double Halbach array energy harvester is $50 \%$ greater than those of the two normal layouts. Compared with the standard Halbach array, the double Halbach array energy harvester has achieved a $700 \%$ increase in average output power. In this case, the width of each magnet in the normal 7 magnet layout is much smaller than the diameter of the big coil. Thus, the magnetic coupling between magnets and the coil is relatively low. In contrast, the double Halbach array has the highest magnetic flux change rate and thus the highest output power.

For the two-coil scenario, it was found that the energy harvester with the double Halbach array has similar output power to that with the normal 7 magnet layout and both are $50 \%$ better than the energy harvester with normal 4 magnet layout. This is because the width of each magnet in the normal 7 magnet layout is similar to the diameter of each small coil and greater magnetic coupling is achieved. This gives it a similar magnetic flux change rate, thus similar output power to that of the double Halbach array.

When comparing the same layout in different coil arrangements, it was found that energy harvesters with one big coil, in most cases, have higher output than those with two small coils because the one big coil has greater total area than the two small coils combined. The only exception is the normal 7 magnet layout where output power is similar for the different coil sets. The reason is that although the big coil has more area, it does not couple with the magnet as efficiently as the two small coils since its diameter is greater than the length of the magnet in the moving coupling direction. 


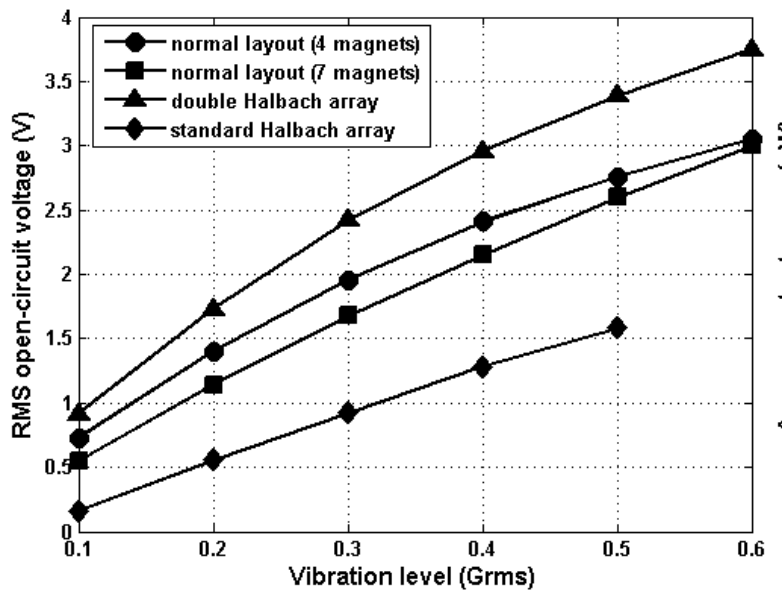

(a)

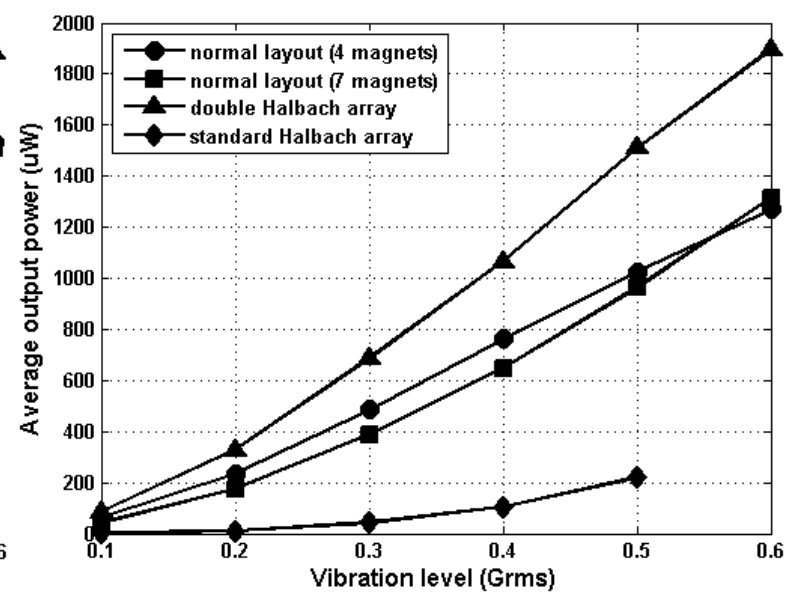

(b)

Figure 13. Comparisons of test results of double Halbach array, normal layouts (4 magnets) and normal layouts (7 magnets) with one big coil (a) open circuit voltage (b) maximum average output power.

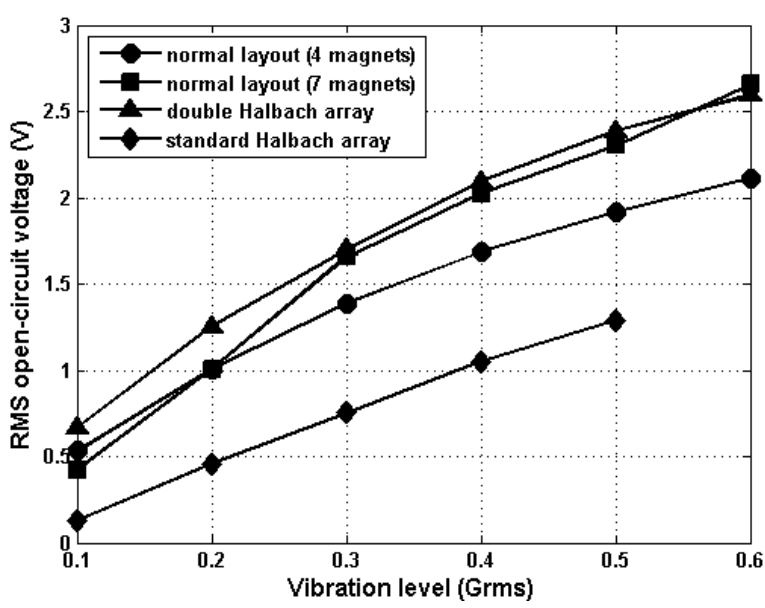

(a)

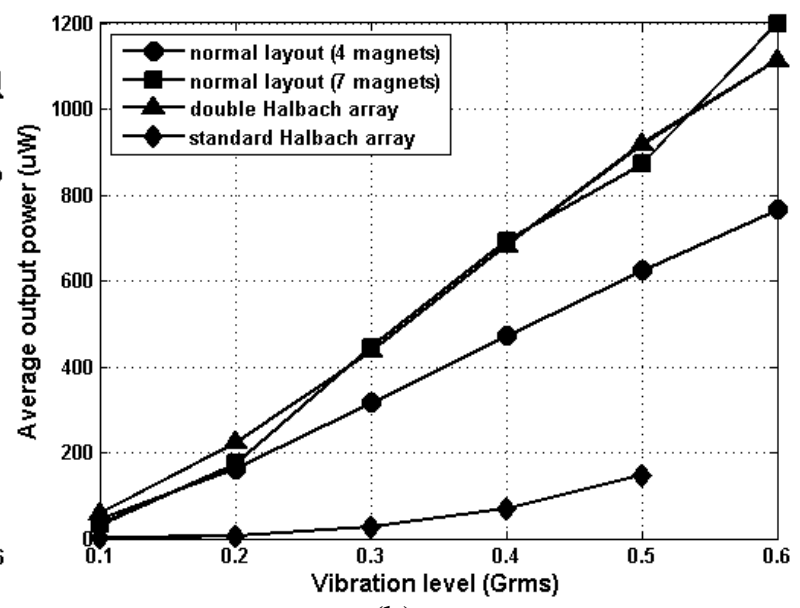

(b)

Figure 14. Comparisons of test results of double Halbach array, normal layouts (4 magnets) and normal layouts (7 magnets) with two small coils (a) open circuit voltage (b) maximum average output power.

\section{Conclusions}

This paper studies two methods of improving the output power of electromagnetic vibration energy harvesters based on Halbach arrays. Based on analysis of the energy harvester with a standard Halbach array, it was found that although the standard Halbach array has higher magnetic field density compared to normal magnet layouts if dimensions of individual magnets are identical, its magnetic flux change rate is not necessarily high due to the existence of transit magnets. Thus, output powers of energy harvesters with the standard Halbach array are not always greater than those with normal magnet layouts.

Two novel Halbach array arrangements, the Halbach array with triangular cross-sectioned magnets and the double Halbach array were proposed in this paper and both successfully minimised the effect of the transit magnets and thus increased the magnetic flux change rate. Theoretically, the magnetic flux change rate can be increased by 1.88 and 2.74 times using the Halbach array with triangular cross-sectioned magnets and the double Halbach array, compared to the previously reported standard Halbach array respectively. Experimentally, it was found that the energy harvester with the Halbach array with triangular cross-sectioned magnets increased the output power by a factor of 3.5 compared to the previous design of standard Halbach array. The energy harvester with the double Halbach array increased the output power by a factor of seven compared to the previous design of standard Halbach 
array. When comparing the new Halbach array arrangements with normal magnetic layouts, it was found that the double Halbach array has higher magnetic flux change rate and thus produces higher output power than the normal magnetic layout while the Halbach array with triangular cross-sectioned magnets does not provide a magnetic flux change rate as high as the normal magnetic layout. Furthermore, this work also highlights the importance of selecting the correct coil dimensions and arrangement. It was found that the radius of the coil has to be similar to the effective length of the magnet to achieve effective electromagnetic coupling.

In conclusion, the Halbach array with an optimised design has the ability to increase electromagnetic coupling within a limited space especially a planar structure compared to the normal magnet layout. Moreover, the fact that the Halbach array concentrates the magnetic field on one side while cancelling the magnetic field on the other side can help further reduce the size of self-powered devices especially those sensitive to magnetic interference. It is worth mentioning that the same phenomenon can be achieved in normal magnet layouts by placing a pole piece behind them and higher magnetic flux change rate can be obtained with this simple structure compared to the Halbach array. However, further research needs to be carried out to study how the thickness of the pole piece will affect the overall performance and, in addition, doing this will increase the overall dimension of the device.

\section{Acknowledgement}

This work is supported by the European Union Seventh Framework Programme (FP7) research project TRIADE (http://triade.wscrp.com/).

\section{References}

[1] Beeby S P, Tudor M J and White N M 2006 Energy harvesting vibration sources for microsystems applications Measurement Science and Technology 17 pp. 175-195

[2] Cao X, Chiang W-J, King Y-C and Lee Y-K 2007 Electromagnetic Energy Harvesting Circuit With Feedforward and Feedback DC-DC PWM Boost Converter for Vibration Power Generator System IEEE Transactions on Power Electronics 22(2) pp. 679-685

[3] Zhu D, Tudor M J and Beeby S P 2010 Strategies for Increasing the Operating Bandwidth of Vibration Energy Harvesters: A Review Measurement Science and Technology 21(2) 022001 (29pp)

[4] Zhu D, Beeby S, Tudor J and Harris N 2012 Vibration energy harvesting using the Halbach array Smart Materials and Structures 21(7) 075020(11pp)

[5] Halbach K 1985 Permanent Magnets for Production and Use of High Energy Particle Beams 8th International Workshop on Rare Earth Magnets and their Applications, Dayton, Ohio, USA, May 1985, Paper No. 11-2

[6] Lee M G, Lee S Q and Gweon D-G 2004 Analysis of Halbach magnet array and its application to linear motor Mechatronics 14 pp. 115-128

[7] Hull J R and Turner L R 2000 Magnetomechanics of Internal-Dipole, Halbach-Array Motor/Generators IEEE Transactions on Magnetics 36(4) pp. 2004-2011

[8] Raich H and Blümler P 2004 Design and construction of a dipolar Halbach array with a homogeneous field from identical bar magnets: NMR Mandhalas Concepts in Magnetic Resonance Part B: Magnetic Resonance Engineering 23B(1) pp. 16-25

[9] Saha C R, Riley P H, Paul J, Yu Z., Jaworski A J and Johnson C M 2012 Halbach array linear alternator for thermo-acoustic engine Sensors and Actuators A 178 pp.179-187

[10] Wang Y-J, Chen C-D, Sung C-K, Li C 2012 Natural frequency self-tuning energy harvester using a circular Halbach array magnetic disk Journal of Intelligent Material Systems and Structures 23(8) pp. 933-943

[11] Gauss meter from Magnet Sales and Service Limited, http://www.magnetsales.co.uk/gaussmeters.htm (accessible on 26 June 2013) 